\title{
Inhibition of Fatty-Acid Amide Hydrolase Accelerates Acquisition and Extinction Rates in a Spatial Memory Task
}

\author{
Stephen A Varvel', Laura E Wise', Floride Niyuhire', Benjamin F Cravatt ${ }^{2}$ and Aron H Lichtman*,' \\ 'Department of Pharmacology and Toxicology, Medical College of Virginia Campus, Virginia Commonwealth University, Richmond, VA, USA; \\ ${ }^{2}$ Skaggs Institute for Chemical Biology and Departments of Cell Biology and Chemistry, The Scripps Research Institute, La Jolla, CA, USA
}

\begin{abstract}
Recent reports have demonstrated that disruption of $\mathrm{CB}_{\text {I }}$ receptor signaling impairs extinction of learned responses in conditioned fear and Morris water maze paradigms. Here, we test the hypothesis that elevating brain levels of the endogenous cannabinoid anandamide through either genetic deletion or pharmacological inhibition of its primary catabolic enzyme fatty-acid amide hydrolase (FAAH) will potentiate extinction in a fixed platform water maze task. FAAH (-/-) mice and mice treated with the FAAH inhibitor OL-I35, did not display any memory impairment or motor disruption, but did exhibit a significant increase in the rate of extinction. Unexpectedly, FAAH-

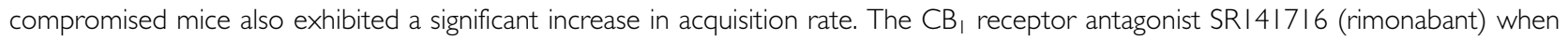
given alone had no effects on acquisition, but disrupted extinction. Additionally, SR I4/7I6 blocked the effects of OL-I35 on both acquisition and extinction. Collectively, these results indicate that endogenous anandamide plays a facilitatory role in extinction through a $\mathrm{CB}_{\text {| }}$ receptor mechanism of action. In contrast, the primary psychoactive constituent of marijuana, $\Delta^{9}$-tetrahydrocannabinol, failed to

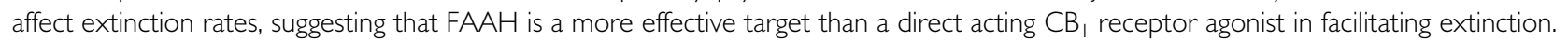
More generally, these findings suggest that FAAH inhibition represents a promising pharmacological approach to treat psychopathologies hallmarked by an inability to extinguish maladaptive behaviors, such as post-traumatic stress syndrome and obsessive-compulsive disorder.
\end{abstract}

Neuropsychopharmacology (2007) 32, 1032-1041. doi:I0.1038/sj.npp. I 301224; published online 18 October 2006

Keywords: extinction; fatty-acid amide hydrolase (FAAH); endogenous cannabinoid; SRI4I7I6 (rimonabant); anandamide; delta9tetrahydrocannabinol (THC)

\section{INTRODUCTION}

Behavioral extinction processes have been studied for decades, and are widely understood to represent a distinct form of inhibitory learning rather than an 'unlearning' of a conditioned response (Rescorla, 2001). Pharmacological manipulation of mechanisms underlying extinction has been suggested as a potential treatment strategy for a variety of behavioral disorders such as specific phobias, post-traumatic stress disorder, and even drug abuse. Extinction processes have been reportedly affected by a variety of pharmacological agents, though the particular mechanisms that trigger the initiation of extinction learning are still largely unclear. For example, NMDA antagonists, voltage-gated calcium channel blockers, benzodiazepines,

*Correspondence: Dr AH Lichtman, Department of Pharmacology and Toxicology, Medical College of Virginia Campus, Virginia Commonwealth University, PO Box 980613, Richmond, VA 23298-0613, USA, Tel: + I 804828 8480, Fax: + I 80482821 17,

E-mail: alichtma@vcu.edu

Received 10 April 2006; revised 29 August 2006; accepted I September 2006

Online publication: 12 September 2006 at http://www.acnp.org/ citations/Npp09|206060232/default.pdf and dopaminergic modulators have all been reported to disrupt extinction, whereas facilitation of extinction has been observed with the partial NMDA agonist D-cycloserine, and the $\mathrm{GABA}_{\mathrm{A}}$ antagonist picrotoxin (Myers and Davis, 2002). Recently, both $\mathrm{CB}_{1}$ receptor $(-I-)$ mice and mice treated with the $\mathrm{CB}_{1}$ antagonist SR141716 (rimonabant) displayed impaired extinction in conditioned fear tests (Marsicano et al, 2002; Suzuki et al, 2004), as well as in the Morris water maze (Varvel et al, 2005a). Taken together, the results of these studies support the hypothesis that the endocannabinoid system participates in processes underlying extinction learning.

Recent years have seen great strides in the characterization of the endocannabinoid system, composed of multiple subtypes of receptor, endogenous ligands, and specific inactivation mechanisms (Lambert and Fowler, 2005). This endogenous system has been implicated in several physiological functions including the modulation of pain (Calignano et al, 1998; Richardson et al, 1998; Walker et al, 1999), feeding (Di Marzo et al, 2001), drug dependence (Ledent et al, 1999; Lichtman et al, 2001; Gonzalez et al, 2002), excitotoxicity (Marsicano et al, 2003), anxiety (Kathuria et al, 2003), depression (Gobbi et al, 2005; Witkin et al, 2005), and cognition (Terranova et al, 1996; Marsicano 
et al, 2002; Varvel et al, 2005a). Of particular interest has been the discovery of fatty-acid amide hydrolase (FAAH), an integral membrane enzyme that is primarily responsible for the degradation of the endocannabinoid anandamide as well as several non-cannabinoid fatty-acid amides (FAAs; (Cravatt et al, 1996)). FAAH (-I-) mice have been shown to possess quantities of anandamide and other FAAs (eg, oleamide, PEA, OEA) in the brain and other areas more than 10 times the levels seen in wild-type mice. Accordingly, FAAH $(-I-)$ mice represent a useful tool to evaluate the physiological function of these lipid signaling molecules (Cravatt et al, 2001; Clement et al, 2003). In addition to displaying dramatically enhanced responses to intraperitoneal injections of anandamide, FAAH $(-/-)$ mice display $\mathrm{CB}_{1}$ receptor mediated hypoalgesic responses to radiant heat and inflammatory stimuli (Cravatt et al, 2001; Lichtman et al, 2004b). In parallel with the transgenic models, several pharmacological inhibitors of FAAH have been developed and shown to elicit cannabinoid-receptor mediated analgesia, notably reversible $\alpha$-ketoheterocyle inhibitors (eg, OL-135), and irreversible carbamate inhibitors (eg, URB-597). URB-597 was shown to elicit a $\mathrm{CB}_{1}$ receptor-mediated hypoalgesic response in the hot plate test (Kathuria et al, 2003) as well as a $\mathrm{CB}_{2}$ receptor mediated anti-edema effect in the carrageenan test (Holt et al, 2005). Similarly, OL-135 produced $\mathrm{CB}_{1}$ receptor mediated hypoalgesic effects in several pain assays including tail immersion, hot plate, and formalin tests (Lichtman et al, 2004a). OL-135 has also been found to block mechanical allodynia in a rat spinal nerve ligation model and this effect was blocked with either the $\mathrm{CB}_{2}$ receptor antagonist SR144528 or naloxone, but not by SR141716 (Chang et al, 2006). On the other hand, only naloxone blocked the allodynia in a mild thermal injury rat model (Chang et al, 2006). Thus, FAAH inhibitors have been demonstrated to decrease pain sensitivity through $\mathrm{CB}_{1}, \mathrm{CB}_{2}$, and opioid receptors.

Despite a growing consensus that the endocannabinoid system modulates cognition, efforts to assess direct acting cannabinoid agonists (eg, WIN55,212-2) on extinction are confounded by the well-documented disruptive effects of these agents on memory and locomotor behavior (Pamplona and Takahashi, 2006). The availability of FAAH (-I-) mice and selective FAAH inhibitors gives us the opportunity to test for the first time whether increasing endogenous anandamide levels will facilitate extinction without impairing memory or causing motor disruption. We have recently reported that FAAH $(-I-)$ mice acquired a fixed-platform water maze task normally, and displayed a slight, but significant, enhancement in the acquisition of a repeated acquisition (ie, working memory) task (Varvel et al, 2005b). Another recent report showed that AM404, which inhibits FAAH and is purportedly an inhibitor of the controversial anandamide uptake transporter (Beltramo et al, 1997; Jarrahian et al, 2000; Glaser et al, 2003; Hillard and Jarrahian, 2005), enhanced the extinction of a fearpotentiated startle response (Chhatwal et al, 2005). Thus the present experiments were conducted to test the hypothesis that elevating endogenous levels of anandamide would enhance extinction. To this end, FAAH $(-I-)$ mice as well as mice treated with OL-135 were evaluated for acquisition and extinction in a fixed-platform water maze task. In addition, SR141716 was employed to assess whether the effects of OL-135 were mediated through the $\mathrm{CB}_{1}$ receptor. In order to compare a direct acting cannabinoid receptor agonist to mice with the FAAH $(-l-)$ mice and OL-135-treated wild-type mice, an additional group of wildtype mice was treated with $\Delta^{9}$-tetrahydrocananbinol (THC), the primary psychoactive constituent present in marijuana, and evaluated for extinction in the Morris water maze extinction task. Here, we report that FAAH-compromised mice, but not THC-treated mice, display a $\mathrm{CB}_{1}$ receptormediated acceleration in extinction rates in the Morris water maze.

\section{MATERIALS AND METHODS}

\section{Subjects}

Male C57BL/6 mice (Jackson Laboratories, Bar Harbor, $\mathrm{ME})$, congenic FAAH $(-/-)$ mice, and offspring (ie, FAAH $+/+$ mice) derived from C57BL/6 breeding pairs from the Virginia Commonwealth University (VCU) vivarium served as subjects. All subjects were 7-12 weeks of age at the start of the study and were housed in a temperature-controlled $\left(20-22^{\circ} \mathrm{C}\right)$ environment, with a 12 -h light/dark cycle. Food and water were available ad libitum in the home cages. All experiments were approved by the Institutional Animal Care and Use Committee at VCU.

\section{Apparatus}

The water maze consisted of a large circular galvanized steel pool ( $1.8 \mathrm{~m}$ diameter, $0.6 \mathrm{~m}$ height). A white platform $(10 \mathrm{~cm}$ diameter) was placed inside, and the tank was filled with water $\left(22^{\circ} \mathrm{C}\right)$ until the top of the platform was submerged $1 \mathrm{~cm}$ below the water's surface. A sufficient amount of white paint (Proline-Latex Flat) was added to make the water opaque and render the platform virtually invisible. An automated tracking system (Columbus Instruments, Columbus, $\mathrm{OH}$ ) analyzed the swim path of each subject and calculated several corresponding dependent measuresescape latencies (the time between being placed in the water and finding the hidden platform), total path lengths, average swim speeds, degree of thigmotaxia (percentage of time spent in periphery of the pool), percent of time spent in the target quadrant, and the number of entries into specified target areas.

\section{Drugs}

THC and SR141716 were provided by the National Institute on Drug Abuse (Bethesda, MD). We selected the reversible inhibitor OL-135 because it is both highly selective for inhibiting FAAH and active in vivo (Lichtman et al, 2004a). In contrast, URB597 binds irreversibly to FAAH and AM404 is not selective. OL-135 was synthesized as described previously (Boger et al, 2005). All drugs were dissolved in a 1:1 mixture of absolute ethanol and alkamuls-620 (Rhone-Poulenc, Princeton, NJ) and diluted with saline to a final ratio of 1:1:18 (ethanol:alkamuls:saline). Drug injections were administered i.p. at an injection volume of $10 \mathrm{ml} / \mathrm{kg}$. 


\section{Procedure}

FAAH $(+/+)$ and $(-/-)$ mice in acquisition and extinction. FAAH $(+/+)$ and $(-/-)$ mice were trained to acquire a fixed-platform water maze task using procedures identical to those previously described (Varvel and Lichtman, 2002; Varvel et al, 2005b), except that a platform location near the 'back' of the tank (ie, furthest from where the experimenter enters and exits the tank area) was chosen. Briefly, each subject was given a single 5-min acclimation trial, in which it was placed in the tank with no platform present. The first minute of this acclimation trial was analyzed separately and used as a baseline measure for the subsequent probe trials evaluated in the extinction phase of the experiment. Mice then received a total of eight acquisition sessions in which the hidden platform remained in a fixed location. All sessions took place between Monday and Friday, with the first acquisition session given on Monday, Tuesday, or Wednesday, but never given on a Thursday or Friday. Consequently, the last acquisition session and the extinction sessions were never given on a Monday. Each session consisted of four 2-min trials with a $10 \mathrm{~min}$ inter-trial interval. Mice were released into the tank from a different position each trial (one of four positions) in an attempt to minimize the use of non-spatial strategies. The day following the final acquisition session the platform was removed from the tank and mice were assessed with a $60 \mathrm{~s}$ probe trial (the first extinction trial). The mice were then given a weekly 60-s trial for a total of 4 weeks. In order to control for the possibility that memory duration is decreased in FAAH $(-I-)$ mice compared with the wild-type mice, a group $(n=6)$ of the FAAH $(-l-)$ were not given the first three extinction trials and were assessed for the first time 3 weeks after completing acquisition training.

OL-135 and SR141716 in acquisition and extinction. Acquisition and extinction procedures used to evaluate the effects of the FAAH inhibitor OL-135 were identical to those described above except that mice received daily one of four drug treatments vehicle + vehicle $(N=10)$, vehicle $+30 \mathrm{mg} / \mathrm{kg}$ OL-135 $(N=11), 3 \mathrm{mg} / \mathrm{kg}$ SR141716 + vehicle $(N=9)$, and $3 \mathrm{mg} / \mathrm{kg} \quad$ SR $141716+30 \mathrm{mg} / \mathrm{kg}$ OL-135 $(N=10), 30 \mathrm{~min}$ before each acquisition session and each extinction trial. In an effort to delineate the effects of FAAH inhibition between acquisition and extinction, a follow-up experiment was conducted in which OL-135 was only administered before each extinction session.

Additional mice were trained to locate the platform using identical procedures as described above except that no drug treatments were administered during acquisition. The day following the last acquisition session mice were treated with vehicle $(N=8)$ or $30 \mathrm{mg} / \mathrm{kg}$ OL-135 $(N=8)$ and evaluated in a $60 \mathrm{~s}$ probe trial, and this process was repeated a week later. In order to distinguish between effects of OL-135 on extinction $v s$ forgetting, a third group $(N=6)$ was simultaneously trained under the same acquisition protocol and the next day was administered $30 \mathrm{mg} / \mathrm{kg}$ OL135 in their home cages (no probe trial). The following week they received a second injection of OL-135 and were assessed with a probe trial.
Effects of THC on extinction. Naive C57BL/6 mice received a 5-min acclimation and eight acquisition sessions as described above. The day following the last acquisition session, each mouse was treated with vehicle or THC (0.1, $0.3,1$, or $10 \mathrm{mg} / \mathrm{kg}$ ) and $30 \mathrm{~min}$ later evaluated in a $60 \mathrm{~s}$ probe trial $(N=7$ or 8 mice per treatment condition). Subjects were then given the same treatment on four additional weekly $60 \mathrm{~s}$ extinction trials.

\section{Statistical Analysis}

The dependent measures of interest for acquisition were escape latency (ie, latency to target) and path length to target. In the extinction tests, we recorded the latencies and path lengths to target as well as the percentage of time that the mice swam in the target quadrant. All experiments were analyzed with two-way (genotype by session or THC by session) or three-way (OL-135 by SR141716 by session or trial) ANOVAs. The Newman-Keuls test was used to compare effects of genotype or drug at each session to the appropriate controls. Additional analyses of the extinction data determined differences from the first extinction trial for each group using Dunnett's test. Significant differences for all analyses were defined as $p<0.05$.

\section{RESULTS}

\section{FAAH (-I-) Mice Exhibit Facilitated Rates of Both Acquisition and Extinction in the Morris Water Maze}

Careful attention was paid to the general behavior of the FAAH $(-I-)$ mice throughout these experiments, and in general they appeared identical to wild-type mice (eg, body weight and home cage behavior). In particular, immediate responses to being placed in the water and overall swimming behavior appeared indistinguishable from wildtype mice. As shown in Figure 1, FAAH $(-/-)$ mice acquired the fixed-platform task more quickly than did

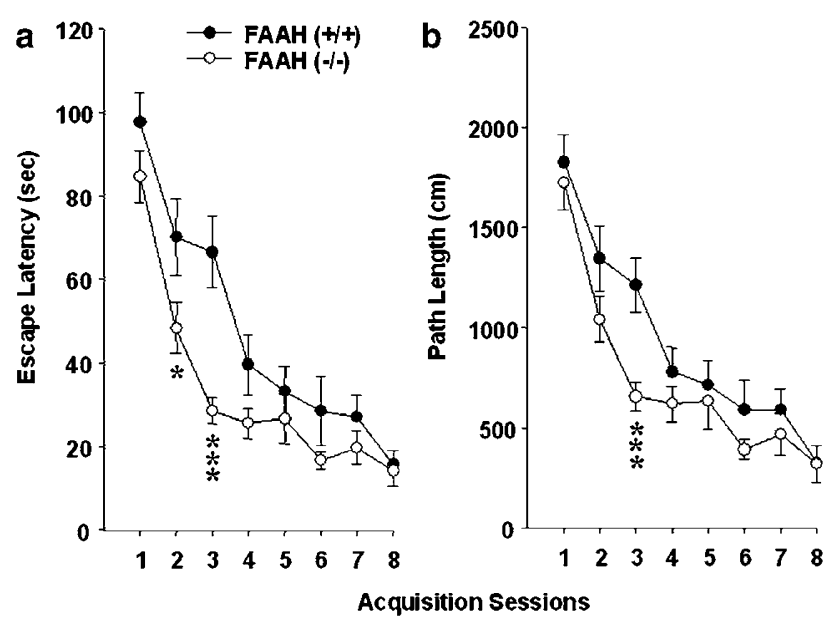

Figure I FAAH $(-/-)$ mice show enhanced acquisition rates in a fixedplatform task. Escape latencies (s) are presented in (a), corresponding path lengths $(\mathrm{cm})$ are shown in (b). Asterisks represent significant differences between genotypes at a given trial, ${ }^{*} p<0.05$, $* * * * 0<0.001$. The data for each session represents the average of four daily trials \pm SEM. $N=11$ $(+/+)$ mice and $16(-/-)$ mice. 
FAAH $(+/+)$ mice. A significant genotype by session interaction was found for escape latencies (Figure 1a, $\mathrm{F}(7,175)=2.3, \quad p<0.05)$, and post hoc comparisons

(Newman-Keuls test) showed that FAAH $(-/-)$ mice found the platform faster than FAAH $(+/+)$ mice on sessions two $(p<0.05)$ and three $(p<0.001)$. A main effect of genotype was also found on path lengths (Figure $1 \mathrm{~b}$, $\mathrm{F}(7,175)=6.9, p<0.05)$, where the distance swum by FAAH $(-/-)$ mice was shorter than FAAH $(+/+)$ mice on session three $(p<0.001)$. An effect of genotype was also found for swim speeds, $\mathrm{F}(7,175)=7.6, p<0.05$, with FAAH $(-/-)$ mice swimming faster on sessions two through four than FAAH $(+/+)$ mice $(p<0.05$, data not shown).

As shown in Figure 2, FAAH $(-/-)$ mice also extinguished this learned response at a quicker rate than FAAH $(+/+)$ mice. Effects of genotype were found for latency to target (Figure 2a, $\mathrm{F}(1,85)=16.0, p<0.001$ ), path length to target (Figure $2 \mathrm{~b}, \mathrm{~F}(1,85)=34.3, p<0.001$ ), and percent of time spent in the target quadrant (Figure $2 c, F(1,85)=5.8$, $p<0.05)$. In contrast, no significant effects were found for swim speed. Post hoc comparisons examining the effect of genotype at each session revealed that FAAH $(-/-)$ mice had longer path lengths to target on the third $(p<0.05)$ and fifth $(p<0.05)$ extinction trials than those of FAAH $(+/+)$ mice. A similar trend was noticed for the latency to target measure, though differences on the third extinction trial only approached significance $(p=0.06)$. The percentage of time spent in the target quadrant was reduced in FAAH $(-/-)$ mice on extinction trials 3 and 4 compared with wild-type mice $(p<0.05)$. A similar pattern of results was obtained when Dunnett's tests were used to identify differences from the first extinction trial for each group. In FAAH $(+/+)$ mice, latencies and path lengths were only different on the fifth (final) trial, whereas the percentage of time in the target quadrant never significantly differed from the first extinction trial. In contrast, FAAH $(-/-)$ mice had longer latencies and less time in the target quadrant on trials 3-5, and higher path lengths on the fourth and fifth trials compared to the first extinction trial. Direct comparisons were made between the FAAH (-I-) group that received three extinction sessions (Group FAAH (-l-) Extinction) and the FAAH $(-/-)$ mice that were not given any extinction trials until 3 weeks after the last day of acquisition (Group FAAH (-/-) No Extinction). Group FAAH $(-/-)$ Extinction had longer latencies (Figure 2a, $p<0.05$ ), longer path lengths (Figure $2 b, p<0.05$ ), and spent less time in the correct quadrant (Figure $2 c, p<0.05$ ) than did group FAAH $(-I-$ ) No Extinction. These findings indicate that that FAAH $(-/-)$ mice exhibited facilitated extinction as opposed to impaired memory duration.

\section{Effects of OL-135 and SR141716 in Acquisition and Extinction Tasks}

The effects of OL-135 and SR141716 pretreatment on escape latency and path length to target on acquisition of the fixed platform task are presented in Figure 3. A main effect on distance swum was found for OL-135 treatment, $\mathrm{F}(1,252)=4.3, p<0.05$, but not for SR141716, $\mathrm{F}(1,252)=0.30, p=0.59$. Post hoc comparisons revealed that path lengths in the OL-135 treatment group were shorter than in the vehicle group on the second and sixth acquisition sessions $(p<0.05)$. Furthermore, the SR141716 + OL-135 group had longer path lengths when compared to the OL-135 group on the second and third acquisition sessions $(p<0.05)$, demonstrating that this effect of OL-135 was prevented by SR141716 treatment. Although a similar trend was observed in the escape latency data (Figure 3a), the main effects of OL-135 $(p=0.11)$ and SR141716 treatment $(p=0.54)$ failed to achieve statistical significance. There were also no effects of OL-135 treatment on swim speeds (data not shown), $\mathrm{F}(1,252)=0.67, p=0.66$. In contrast, SR141716 significantly increased speeds,
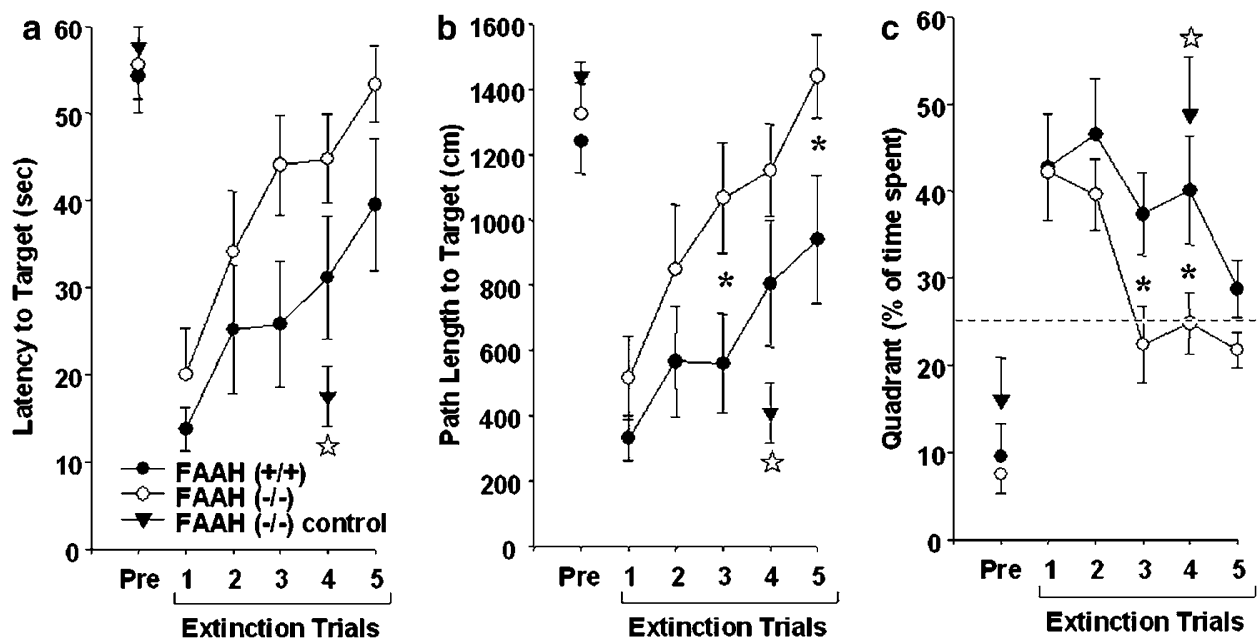

Figure 2 FAAH $(-/-)$ mice show enhanced extinction compared to FAAH $(+/+)$ mice. Latencies $(s)$ to reach where the platform had been located are presented in (a), corresponding path lengths to target $(\mathrm{cm})$ are shown in (b), and the percentage of time spent in the target quadrant is presented in (c) (dotted line from the $25 \%$ point of the ordinate spanning the width of the abscissa indicates chance performance). The first probe trial was conducted before acquisition (baseline), subsequent extinction trials were conducted the day after the last acquisition session and once per week after that. Asterisks represent significant differences between genotypes at a given trial, $* 2<0.05$, stars $(\xi)$ ) represent significant differences between FAAH $(-/-)$ mice and the FAAH $(-/-)$ control group on extinction trial $4, p<0.05$. All data are represented as mean \pm SEM. $N=9(+/+)$ mice, $10(-/-)$ mice, and $6(-/-)$ control mice. 
$\mathrm{F}(1,252)=12.3, p<0.01$, as the SR141716 group swam faster than the vehicle group on the first, third, and fourth sessions $(p<0.05)$.

Results from the extinction experiment showed that OL-135 also enhanced extinction rates, and that SR141716 blocked these effects and disrupted extinction when given alone for each of the dependent measures (ie, latency, path length, and quadrant data; see Figure 4). A three-way

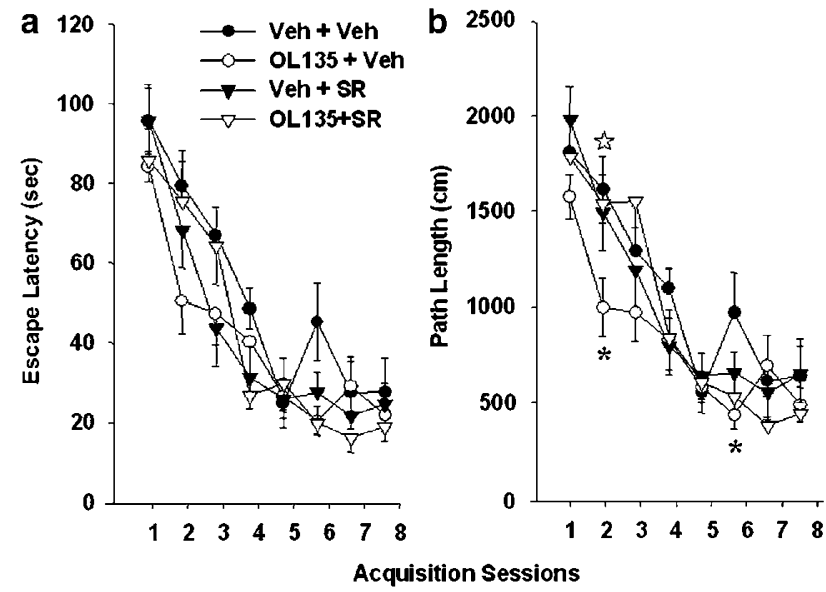

Figure 3 Effects of daily treatments with $30 \mathrm{mg} / \mathrm{kg}$ OL-135, $3 \mathrm{mg} / \mathrm{kg}$ SR $|4| 7 \mid 6$, or the combination of both on acquisition of a standard water maze task. Escape latencies (s) are presented in (a), corresponding path lengths $(\mathrm{cm})$ in (b). The data for each session represents the average of four daily trials \pm SEM. Asterisks represent significant differences between the veh + veh group and the OL-135+veh group, $* p<0.05$. Stars ( 25 ) represent significant differences between the OL-135+veh and OL-135+ SR $14|7| 6$ groups, $p<0.05 . N=7-1 \mid$ mice per group.
ANOVA conducted on the extinction trial latencies to target (Figure 4a) revealed main effects of OL-135, $\mathrm{F}(1,192)=5.7$, $p<0.05, \quad$ SR141716, $\mathrm{F}(1,192)=13.0, \quad p<0.01$, and trial, $\mathrm{F}(6,192)=18.4, p<0.001$. However, no significant effects were found for swim speed. Post hoc comparisons revealed that latency to target was longer in the OL-135 group compared to vehicle on the second extinction trial $(p<0.05)$, and that this effect was prevented by cotreatment with SR141716 $(p<0.05)$. SR141716 by itself produced opposite effects of OL-135, as this group had faster latencies to target compared to vehicle on the fourth extinction trial $(p<0.05)$. Similar results were found for path length to target data (Figure $4 \mathrm{~b}$ ), where main effects were found for OL-135, $\mathrm{F}(1,192)=4.2, p<0.05$, and SR141716, $F(1,192)=5.8, p<0.05$. OL-135-treated mice had longer path lengths on the second extinction trial compared to vehicle-treated $(p<0.05)$ or OL-135+ SR141716-treated mice $(p<0.05)$. Analysis of the percent of time spent in the target quadrant (see Figure 4c) failed to reveal significant effects of OL-135, $\mathrm{F}(1,192)=2.0, p=0.17$, though there were significant effects of SR141716, $\mathrm{F}(1,192)=6.7, \quad p<0.05, \quad$ and session, $\mathrm{F}(6,192)=14.3$, $p<0.001$. Dunnett's tests conducted for each group to identify differences from the first extinction trial found that the vehicle-treated group had increases in both latency to target and path length to target on trials 4-6, and spent less time in the target quadrant only on the fourth trial. Supporting the interpretation of facilitated extinction, the OL-135-treated group displayed significantly longer latencies and longer path lengths on trials $2-6$ than on trial 1. Similarly, these mice spent less time in the target quadrant on trials $4-6$ than trial 1 . The opposite effect was observed in the SR141716-treated mice, as no extinction trials differed from the first on any
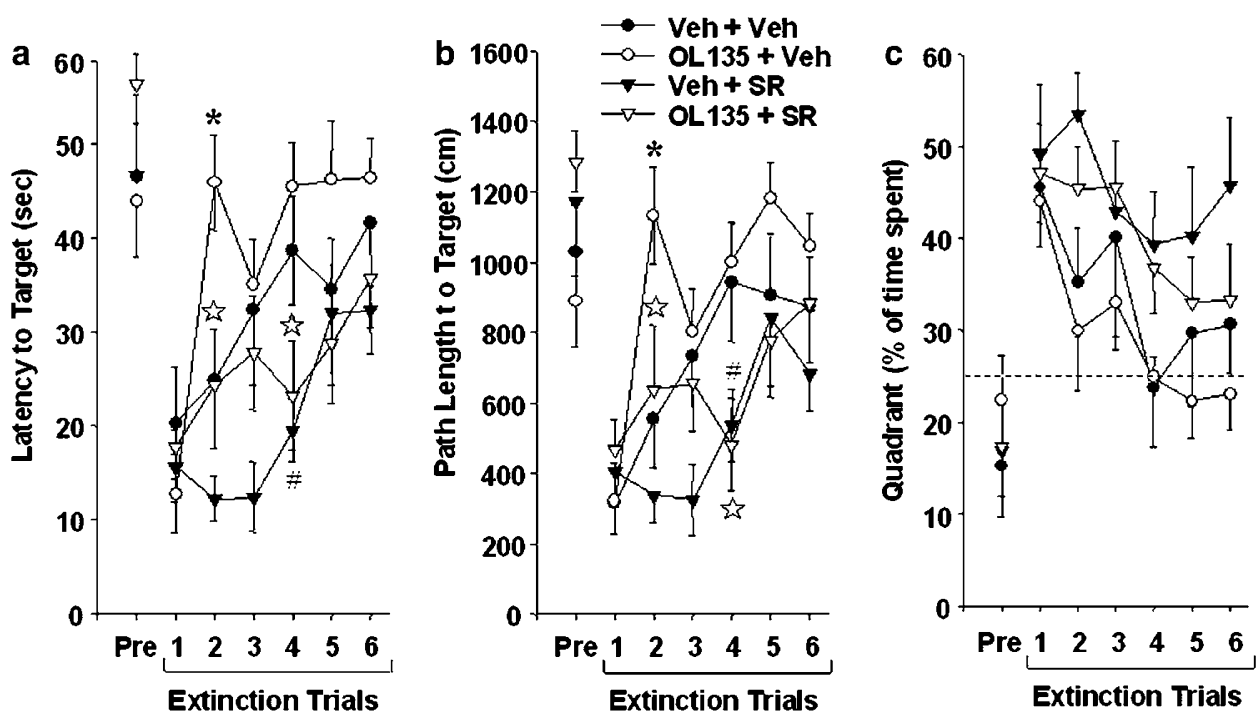

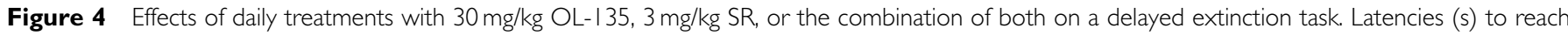
where the platform had been located are presented in the (a), corresponding path lengths to target are shown in (b), and the percentage of time spent in the correct quadrant is presented in (c) (dotted line from the $25 \%$ point of the ordinate spanning the width of the abscissa indicates chance performance). Asterisks represent significant differences between the veh + veh and OL- I35 + veh, * $p<0.05$. Stars ( $)$ ) represent significant differences between the OL$135+$ veh and OL- $135+$ SR 141716 groups, $p<0.05$. Pound signs (\#) represent differences between the veh + veh and veh + SR I $417 \mid 6$ groups, $p<0.05$ Post hoc comparison of each extinction trial compared to the first extinction trial for each respective group can be found in the text. All data are represented as mean \pm SEM. $N=7-1$ I mice per group. 
measure. The OL-135+SR141716 group resembled the SR141716 alone group in that the latency to target measure for both of these groups was significantly increased only on the sixth extinction trial, and no differences were observed on path lengths or time spent in the target quadrant.

\section{OL-135 Given Only before Extinction Trials}

The effects of OL-135 given before the extinction probes and not during acquisition are presented in Figure 5, and swim traces of a representative mouse from each treatment group are shown in Figure 6. Escape latencies (Figure 5a, $\mathrm{F}(1,28)=17.9, \quad p<0.001$ ) path lengths (Figure 5b, $\mathrm{F}(1,28)=10.0, p<0.01)$ and time in the correct quadrant, $\mathrm{F}(1,28)=6.9, p<0.05$, were all affected by OL-135 treatment. Post hoc comparisons revealed that the OL-135treated group had longer latencies and path lengths on the third probe (ie, second extinction trial) than did vehicletreated mice $(p<0.05)$, however, the quadrant data failed to achieve significance on this second extinction probe. Further analysis of the quadrant data showed that whereas there was a significant decrease in the time spent in the target quadrant between the first and second extinction trial for the OL-135 group $(p<0.05)$, the vehicle group did not change. Direct comparisons between the OL-135-treated group that received a previous extinction session and the OL-135-treated group that received only a single probe trial 1 week after acquisition revealed differences in latency to the target location (Figure $5 \mathrm{a}, p<0.05$ ) and path lengths (Figure 5b, $p<0.05$ ), but not time spent in the target quadrant, indicating that OL-135 specifically facilitated extinction rates and did not have any apparent effects on forgetting following the passage of time.

\section{THC Fails to Affect Extinction}

As shown in Figure 7, THC failed to have any significant effects on the latency to target location, path length to target location, and time spent in the quadrant that the target had previously been located. For each dependent measure, a significant repeated measures effect was found for probe session $(p<0.001)$, indicating that the groups underwent extinction across the 5 weekly probe trials. However, the main effect of THC as well as the THC by probe interaction failed to achieve significance for each of the three dependent measures.

\section{DISCUSSION}

The results of the present study demonstrate for the first time that elevating brain levels of FAAs, including the endogenous cannabinoid anandamide, via either genetic deletion or pharmacological inhibition of FAAH facilitated the extinction of a spatial memory task. These findings support the hypothesis that the endocannabinoid system plays an important role in modulating extinction learning (Marsicano et al, 2002; Suzuki et al, 2004; Varvel et al, 2005a). In contrast, a wide dose range of THC failed to have any significant effects on extinction. An unexpected finding was that both the FAAH $(-I-)$ mice and mice treated with the FAAH inhibitor OL-135 acquired the hidden platform task faster than their respective controls. The effects of OL-135 on both acquisition and extinction were reversed by SR141716, implicating a $\mathrm{CB}_{1}$ receptor mechanism of action. Importantly, the effects of FAAH deletion and FAAH inhibition on extinction were distinguished from effects on forgetting, as the experience of non-reinforced trials was necessary to observe this effect.
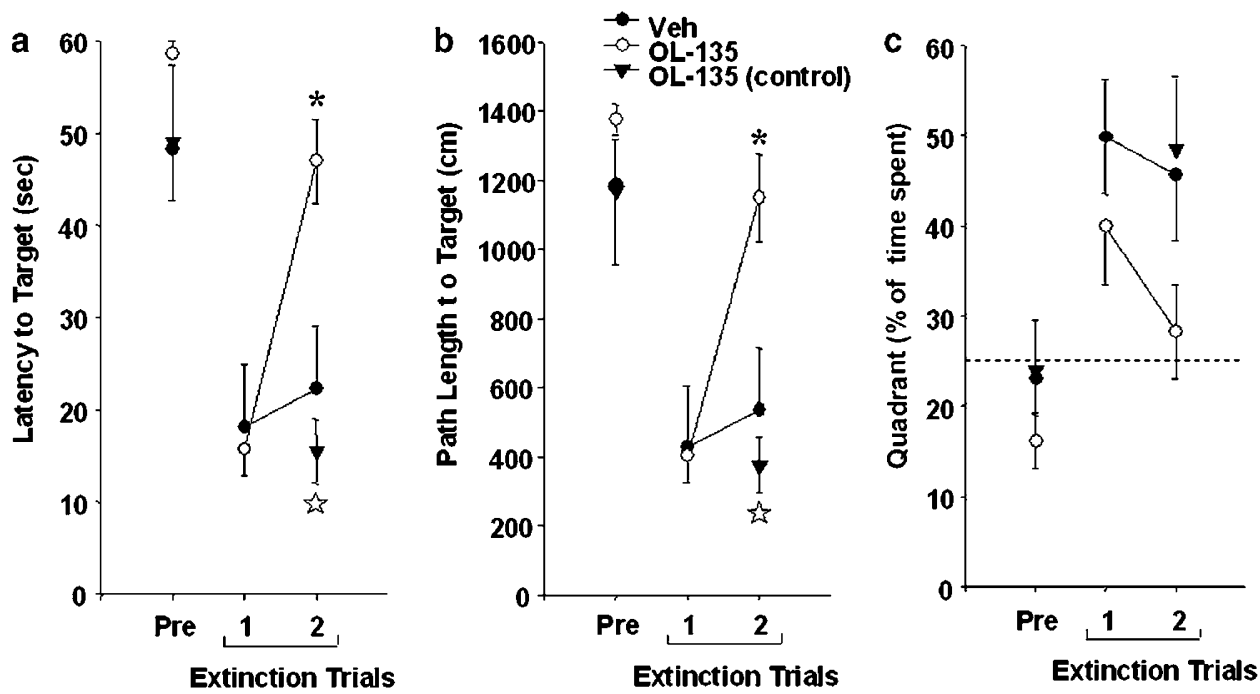

Figure 5 OL-135 (30 mg/kg) accelerates extinction rate when administered only before each extinction trial. In contrast, a control group that received OL- 135 in their home cages the day after acquisition training but were not given their first probe (ie, extinction) trial until 8 days later exhibited near perfect performance, indicating that OL-I 35 per se did not simply result in forgetting. Latencies (ss) to enter the location where the platform had been previously situated are presented in the (a), corresponding path lengths to target are shown in (b), and the percentage of time spent in the correct quadrant is presented in (c) (dotted line from the $25 \%$ point of the ordinate spanning the width of the abscissa indicates chance performance). Asterisks $(*)$ represent significant differences $(p<0.05)$ between vehicle $(N=8$ mice) and $O L-135-(N=8$ mice) treated groups. Stars ( $\langle$ ) represent significant differences $(p<0.05)$ between the OL- I35-treated group that received one extinction trial and the OL-135-treated group that received two extinction trials. All data are represented as mean \pm SEM $(N=6$ mice). 


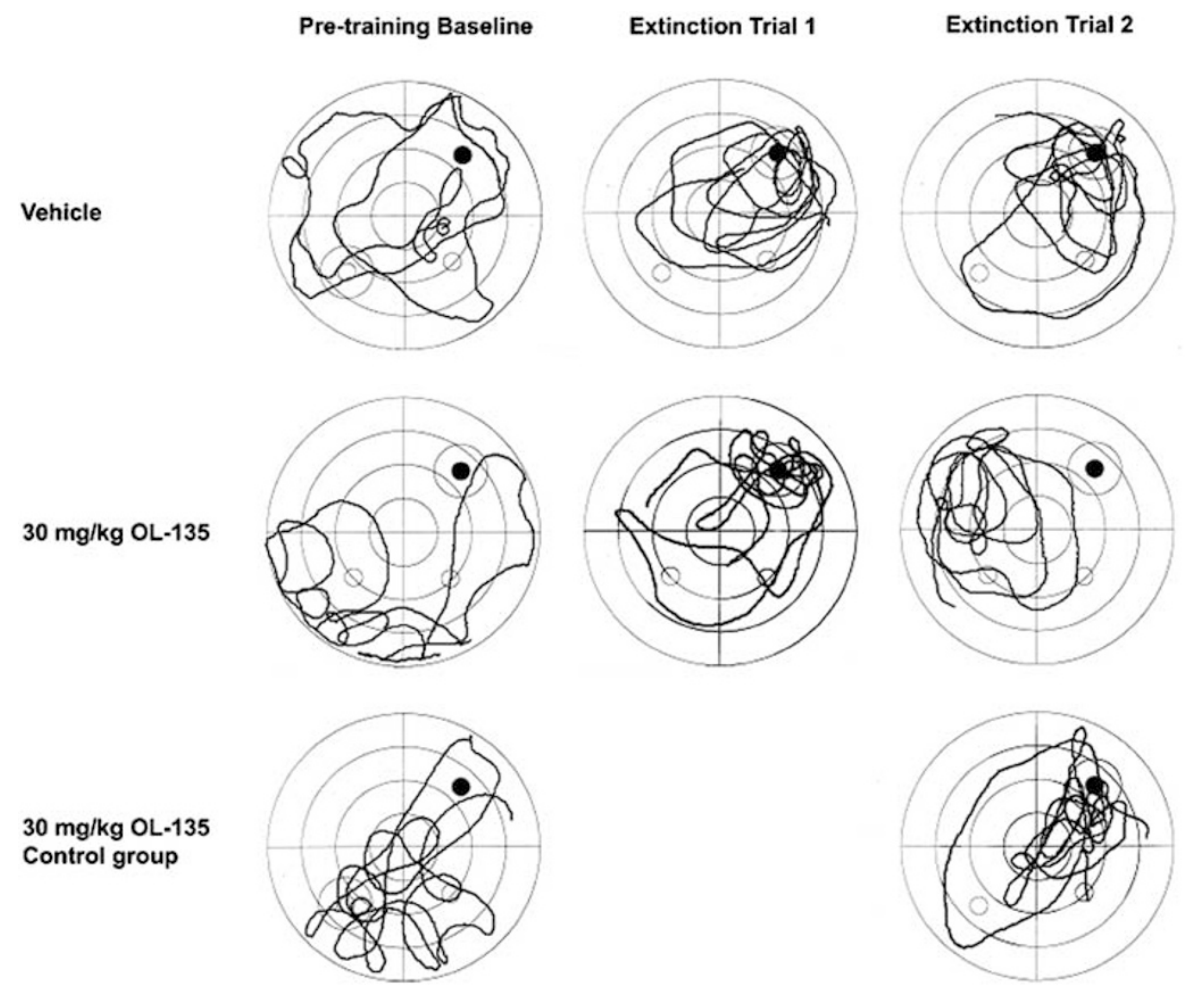

Figure 6 Swim traces of representative mice in each treatment condition from the experiment presented in Figure 5.

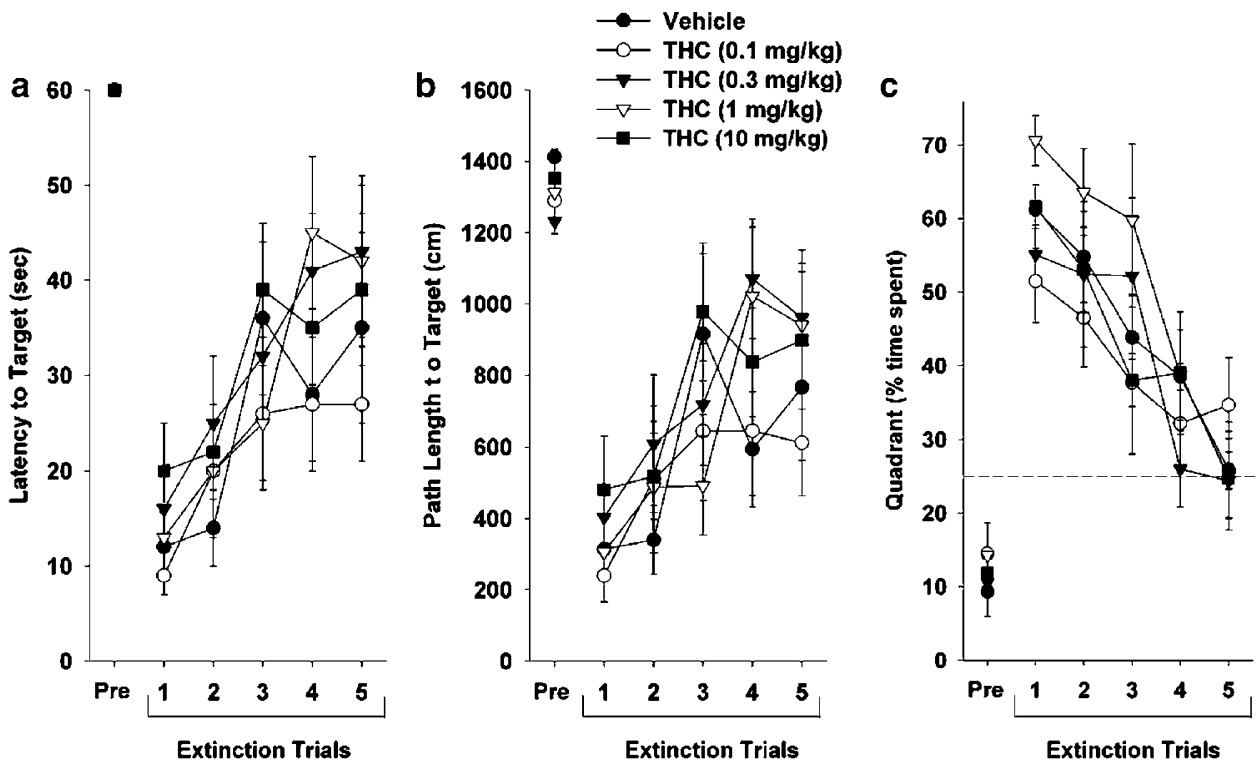

Figure 7 Repeated i.p. injections of THC given 30 min before each extinction probe trial failed to affect latency to target (a), path length to target (b), and percentage of time spent in the target quadrant (c). The dotted line from the $25 \%$ point of the ordinate spanning the width of the abscissa in (c) indicates chance performance). All data are represented as mean $\pm \operatorname{SEM}(N=7-8$ mice/group).

The most prominent finding of these experiments is that FAAH $(-/-)$ mice and C57BL/6 mice treated with OL-135 exhibited accelerated extinction rates of learned spatial behavior compared to their respective controls. This effect was dramatically evident on the second extinction trial in the OL-135-treated mice, suggesting that a single extinction trial in the presence of elevated FAAs was sufficient to extinguish the learned response. The initial FAAH $(-/-)$ and OL-135 experiments were incapable of distinguishing whether it was necessary that FAAH be suppressed during both acquisition and extinction phases because anandamide and other FAAs levels were elevated throughout both phases. Consequently, we evaluated whether OL-135 administered only before each extinction trial would facilitate 
this process, and showed that elevated levels of FAAs were only required during the extinction trials themselves. This effect is consistent with a recent report in which AM404, a purported inhibitor of the putative anandamide transporter that also inhibits FAAH (Beltramo et al, 1997; Jarrahian et al, 2000; Glaser et al, 2003; Hillard and Jarrahian, 2005) potentiated extinction of a conditioned fear response (startle) in rats (Chhatwal et al, 2005).

In the present study, OL-135-induced acceleration of extinction was prevented by pretreatment with SR141716, suggesting a $\mathrm{CB}_{1}$ receptor mechanism of action. However this interpretation is somewhat limited by the fact that SR141716 given alone delayed extinction. As previously described in a similar water maze task (Varvel et al, 2005a) and in conditioned fear paradigms (Marsicano et al, 2002; Suzuki et al, 2004; Chhatwal et al, 2005), SR141716 robustly attenuated extinction in the present experiment. The effect of SR141716 presented here replicates our previously published report, with some minor procedural differences such as the weekly extinction trials (as opposed to bi-weekly trials) and the inclusion of the quadrant data.

Taken together, the ability to increase or decrease extinction rates, respectively, through the enhancement or attenuation of endocannabinoid receptor signaling strongly suggests a physiological role for the endocannabinoid system in modulating extinction. It is noteworthy that Marsicano et al (2002) found elevated levels of anandamide and $2-\mathrm{AG}$ in the amygdala of mice undergoing extinction in the conditioned freezing task, suggesting that experiencing non-reinforced trials increases the production and/or release of endocannabinoids. Consistent with the notion that the endocannabinoid system plays an integral role in mnemonic processes, electrophysiological studies have demonstrated that endocannabinoids are required for certain forms of synaptic plasticity, such as long-term depression in several brain regions (Gerdeman et al, 2002; Hoffman et al, 2003; Huang et al, 2003; Robbe et al, 2003).

The facilitation of acquisition and extinction in FAAH $(-/-)$ mice and OL-135-treated mice reported here provides a dramatic example of how manipulations that elevate endogenous compounds can lead to qualitatively different results compared to exogenous administration of direct-acting receptor agonists. THC $(0.1-10 \mathrm{mg} / \mathrm{kg})$ failed to affect performance during the first post-acquisition probe trial. Similarly, we have previously reported that mice that are given extensive training in a fixed platform task are relatively impervious to the memory disruptive effects of THC as well as the muscarinic antagonists scopolamine (Varvel et al, 2001). On the other hand, cannabinoid agonists have been demonstrated to impair acquisition of the fixed-platform water maze task (Ferrari et al, 1999; da Silva and Takahashi, 2002). Similarly, we have found that cannabinoid receptor agonists consistently impair performance in a working memory version of the Morris water maze in which the location of the platform is changed before each session (Varvel et al, 2001, 2005b; Varvel and Lichtman, 2002). However, here we show that THC given before each of the five post-acquisition probe trials failed to have any significant effects on extinction. Thus, the stimulation of $\mathrm{CB}_{1}$ receptors through either direct-acting agonists or elevating endogenous levels of anandamide leads to a strikingly distinct pattern of effects in this spatial task.

The results with the FAAH $(-I-)$ mice reported here contrast with previous findings from our lab in which FAAH (-I-) mice acquired the fixed platform task identically to their wild-type control littermates (Varvel et al, 2005b). A potentially relevant procedural difference between our current and previous studies is the location of the hidden platform. In the previous studies, the hidden platform was arbitrarily placed towards the front of the tank (ie, closest to where the experimenter entered and exited the maze enclosure). However, in the present study, the platform was moved to the 'back' of the tank (ie, furthest from where the experimenter entered and exited the maze enclosure), in order to utilize the computer software for quantifying the percentage of time subjects spent in the target quadrant. The new platform location appears to have led to a slower acquisition rate in control animals compared to the rather steep curves generated when the platform was located in the 'front' of the tank. Consequently, the rapid rate of acquisition in our previous studies precluded the possibility of detecting any enhancement in FAAH $(-/-)$ mice. However, by changing the platform location in the present study, we appear to have inadvertently unmasked a phenotypic enhancement in acquisition. A similar modest enhancement of acquisition was observed in OL-135-treated mice. The finding that SR141716 blocked this effect indicates a $\mathrm{CB}_{1}$ receptor mechanism, and accordingly implicates the involvement of anandamide, as anandamide is the only known FAAH substrate with relevant $\mathrm{CB}_{1}$ receptor agonist activity.

The enhanced acquisition rate caused by stimulating the endocannabinoid system was an unexpected and unique observation. Interestingly, recent work has shown that mature, but not young, $\mathrm{CB}_{1}$ receptor $(-/-)$ mice display cognitive deficits resembling those seen in aged $(+/+)$ mice, suggesting that the endocannabinoid system may serve to protect against age-related cognitive decline (Bilkei-Gorzo et al, 2005). Collectively, these results raise the provocative possibility that facilitation of the endocannabinoid system may represent a novel target for nootropic activity. Alternatively, the enhanced acquisition rates seen in the present study may be related to alterations in emotional responding, rather than an effect on cognition. Specifically, as carbamate FAAH inhibitors have been shown to possess anxiolytic activity in the elevated zero maze and isolation-induced vocalization assays in rats (Kathuria et al, 2003), it is plausible that the enhanced acquisition in the present study was a result of reduced stress during the initial sessions. Nonetheless, the lack of effect on thigmotaxia (considered a measure of anxiety) in the present study (data not shown) is not consistent with explanations of anxiolysis.

A pharmacological approach to facilitate extinction learning has the potential to provide clinical benefits for the treatment of phobias and related anxiety disorders. For example, post-traumatic stress disorder is believed to be due to impaired extinction of normal responses to trauma (eg, re-experiencing, avoidance, and hyper-arousal), which continue to be elicited by trauma-related cues long after the traumatic event (Rothbaum and Davis, 2003). Support of this extinction deficit model of PTSD comes from clinical 
work suggesting that exposure of the patient to traumarelated cues in a safe environment (ie, extinction training) is the most empirically validated treatment of PTSD (Rothbaum et al, 2000). The concept of combining pharmacological strategies with behavioral exposure therapy to treat anxiety disorders has recently been validated by a study that demonstrated superior benefits of combined treatment with the NMDA partial agonist D-cycloserine, which has been shown to facilitate extinction of conditioned fear in rats (Walker et al, 2002), with psychotherapy to treat patients diagnosed with acrophobia (Ressler et al, 2004). The lack of any apparent untoward phenotypes in FAAH $(-/-)$ mice or animals treated with FAAH inhibitors further supports the notion that FAAH inhibition represents a particularly desirable therapeutic target.

In conclusion, the results of the present study indicate that the endogenous cannabinoid system may play a relevant role in facilitating extinction processes in the Morris water maze task. These processes can be enhanced or retarded through blockade of FAAH or the disruption of $\mathrm{CB}_{1}$ receptors, respectively. The pharmacological potentiation of extinction learning by inhibitors of FAAH may prove particularly useful in conjunction with behavioral exposure therapy in the treatment of a variety of psychopathologies such as post-traumatic stress syndrome, which are hallmarked by an inability to extinguish maladaptive behaviors.

\section{ACKNOWLEDGEMENTS}

This work was supported by NIDA Grants DA015683, DA017259, DA005274, and T23DA07027. The authors are very grateful for the guidance and suggestions of Dr Billy R Martin.

\section{REFERENCES}

Beltramo M, di Tomaso E, Piomelli D (1997). Inhibition of anandamide hydrolysis in rat brain tissue by (E)-6-(bromomethylene) tetrahydro-3-(1-naphthalenyl)-2H-pyran-2-one. FEBS Lett 403: 263-267.

Bilkei-Gorzo A, Racz I, Valverde O, Otto M, Michel K, Sastre M et al (2005). Early age-related cognitive impairment in mice lacking cannabinoid CB1 receptors. Proc Natl Acad Sci USA 102: 15670-15675.

Boger DL, Miyauchi H, Du W, Hardouin C, Fecik RA, Cheng H et al (2005). Discovery of a potent, selective, and efficacious class of reversible alpha-ketoheterocycle inhibitors of fatty acid amide hydrolase effective as analgesics. J Med Chem 48: 1849-1856.

Calignano A, La Rana G, Giuffrida A, Piomelli D (1998). Control of pain initiation by endogenous cannabinoids. Nature 394: 277-281.

Chang L, Luo L, Palmer JA, Sutton S, Wilson SJ, Barbier AJ et al (2006). Inhibition of fatty acid amide hydrolase produces analgesia by multiple mechanisms. Br J Pharmacol 47: 1812-1822.

Chhatwal JP, Davis M, Maguschak KA, Ressler KJ (2005). Enhancing cannabinoid neurotransmission augments the extinction of conditioned fear. Neuropsychopharmacology 30: 516-524.

Clement AB, Hawkins EG, Lichtman AH, Cravatt BF (2003). Increased seizure susceptibility and proconvulsant activity of anandamide in mice lacking fatty acid amide hydrolase. J Neurosci 23: 3916-3923.
Cravatt BF, Demarest K, Patricelli MP, Bracey MH, Giang DK, Martin BR et al (2001). Supersensitivity to anandamide and enhanced endogenous cannabinoid signaling in mice lacking fatty acid amide hydrolase. Proc Natl Acad Sci USA 98: 9371-9376.

Cravatt BF, Giang DK, Mayfield SP, Boger DL, Lerner RA, Gilula NB (1996). Molecular characterization of an enzyme that degrades neuromodulatory fatty-acid amides. Nature 384: 83-87.

da Silva GE, Takahashi RN (2002). SR 141716A prevents delta 9-tetrahydrocannabinol-induced spatial learning deficit in a Morris-type water maze in mice. Prog Neuropsychopharmacol Biol Psychiatry 26: 321-325.

Di Marzo V, Goparaju SK, Wang L, Liu J, Batkai S, Jarai Z et al (2001). Leptin-regulated endocannabinoids are involved in maintaining food intake. Nature 410: 822-825.

Ferrari F, Ottani A, Vivoli R, Giuliani D (1999). Learning impairment produced in rats by the cannabinoid agonist HU 210 in a water-maze task. Pharmacol Biochem Behav 64: 555-561.

Gerdeman GL, Ronesi J, Lovinger DM (2002). Postsynaptic endocannabinoid release is critical to long-term depression in the striatum. Nat Neurosci 5: 446-451.

Glaser ST, Abumrad NA, Fatade F, Kaczocha M, Studholme KM, Deutsch DG (2003). Evidence against the presence of an anandamide transporter. Proc Natl Acad Sci USA 100: $4269-4274$.

Gobbi G, Bambico FR, Mangieri R, Bortolato M, Campolongo P, Solinas $\mathrm{M}$ et al (2005). Antidepressant-like activity and modulation of brain monoaminergic transmission by blockade of anandamide hydrolysis. Proc Natl Acad Sci USA 102: $18620-18625$.

Gonzalez S, Cascio MG, Fernandez-Ruiz J, Fezza F, Di Marzo V, Ramos JA (2002). Changes in endocannabinoid contents in the brain of rats chronically exposed to nicotine, ethanol or cocaine. Brain Res 954: 73-81.

Hillard CJ, Jarrahian A (2005). Accumulation of anandamide: Evidence for cellular diversity. Neuropharmacology 48 1072-1078.

Hoffman AF, Oz M, Caulder T, Lupica CR (2003). Functional tolerance and blockade of long-term depression at synapses in the nucleus accumbens after chronic cannabinoid exposure. J Neurosci 23: 4815-4820.

Holt S, Comelli F, Costa B, Fowler CJ (2005). Inhibitors of fatty acid amide hydrolase reduce carrageenan-induced hind paw inflammation in pentobarbital-treated mice: comparison with indomethacin and possible involvement of cannabinoid receptors. Br J Pharmacol 146: 467-476.

Huang YC, Wang SJ, Chiou LC, Gean PW (2003). Mediation of amphetamine-induced long-term depression of synaptic transmission by CB1 cannabinoid receptors in the rat amygdala. J Neurosci 23: 10311-10320.

Jarrahian A, Manna S, Edgemond WS, Campbell WB, Hillard CJ (2000). Structure-activity relationships among N-arachidonylethanolamine (Anandamide) head group analogues for the anandamide transporter. $J$ Neurochem 74: 2597-2606.

Kathuria S, Gaetani S, Fegley D, Valino F, Duranti A, Tontini A et al (2003). Modulation of anxiety through blockade of anandamide hydrolysis. Nat Med 9: 76-81.

Lambert DM, Fowler CJ (2005). The endocannabinoid system: drug targets, lead compounds, and potential therapeutic applications. J Med Chem 48: 5059-5087.

Ledent C, Valverde O, Cossu G, Petitet F, Aubert JF, Beslot F et al (1999). Unresponsiveness to cannabinoids and reduced addictive effects of opiates in CB1 receptor knockout mice. Science 283: 401-404.

Lichtman AH, Leung D, Shelton CC, Saghatelian A, Hardouin C, Boger DL et al (2004a). Reversible inhibitors of fatty acid amide hydrolase that promote analgesia: evidence for an unprece- 
dented combination of potency and selectivity. J Pharmacol Exp Ther 311: 441-448.

Lichtman AH, Sheikh SM, Loh HH, Martin BR (2001). Opioid and cannabinoid modulation of precipitated withdrawal in delta(9)-tetrahydrocannabinol and morphine-dependent mice. J Pharmacol Exp Ther 298: 1007-1014.

Lichtman AH, Shelton CC, Advani T, Cravatt BF (2004b). Mice lacking fatty acid amide hydrolase exhibit a cannabinoid receptor-mediated phenotypic hypoalgesia. Pain 109: 319-327.

Marsicano G, Goodenough S, Monory K, Hermann H, Eder M, Cannich A et al (2003). CB1 cannabinoid receptors and ondemand defense against excitotoxicity. Science 302: 84-88.

Marsicano G, Wotjak CT, Azad SC, Bisogno T, Rammes G, Cascio MG et al (2002). The endogenous cannabinoid system controls extinction of aversive memories. Nature 418: 530-534.

Myers KM, Davis M (2002). Behavioral and neural analysis of extinction. Neuron 36: 567-584.

Pamplona FA, Takahashi RN (2006). WIN 55212-2 impairs contextual fear conditioning through the activation of CB1 cannabinoid receptors. Neurosci Lett 397: 88-92.

Rescorla RA (2001). Experimental extinction. In: Mowrer RR, Klein SB (eds). Handbook of Contemporary Learning Theories. Erlbaum: Mahawah, NJ. pp 119-155.

Ressler KJ, Rothbaum BO, Tannenbaum L, Anderson P, Graap K, Zimand $\mathrm{E}$ et al (2004). Cognitive enhancers as adjuncts to psychotherapy: use of D-cycloserine in phobic individuals to facilitate extinction of fear. Arch Gen Psychiatry 61: 1136-1144.

Richardson JD, Aanonsen L, Hargreaves KM (1998). Hypoactivity of the spinal cannabinoid system results in NMDA-dependent hyperalgesia. J Neurosci 18: 451-457.

Robbe D, Alonso G, Manzoni OJ (2003). Exogenous and endogenous cannabinoids control synaptic transmission in mice nucleus accumbens. Ann NY Acad Sci 1003: 212-225.

Rothbaum BO, Davis M (2003). Applying learning principles to the treatment of post-trauma reactions. Ann NY Acad Sci 1008: $112-121$.
Rothbaum BO, Meadows EA, Resick P, Foy DW (2000). Cognitivebehavioral therapy. In: Foa EB, Friedman M, Keane T (eds) Effective Treatments for Posttraumatic Stress Disorder: Practice guidelines from the International Society for Traumatic Stress Studies. Guilford Press: New York. pp 60-83.

Suzuki A, Josselyn SA, Frankland PW, Masushige S, Silva AJ, Kida S (2004). Memory reconsolidation and extinction have distinct temporal and biochemical signatures. J Neurosci 24: 4787-4795.

Terranova JP, Storme JJ, Lafon N, Perio A, Rinaldi-Carmona M, Le Fur $G$ et al (1996). Improvement of memory in rodents by the selective CB1 cannabinoid receptor antagonist, SR 141716. Psychopharmacol 126: 165-172.

Varvel SA, Anum EA, Lichtman AH (2005a). Disruption of CB(1) receptor signaling impairs extinction of spatial memory in mice. Psychopharmacology (Berlin) 179: 863-872.

Varvel SA, Cravatt BF, Engram AE, Lichtman AH (2005b). FAAH $(-/-)$ mice exhibit an increased sensitivity to the disruptive effects of anandamide or oleamide in a working memory water maze task. J Pharmacol Exp Ther 179: 863-872.

Varvel SA, Hamm RJ, Martin BR, Lichtman AH (2001). Differential effects of delta9-THC on spatial reference and working memory in mice. Psychopharmacology (Berlin) 157: 142-150.

Varvel SA, Lichtman AH (2002). Evaluation of CB1 receptor knockout mice in the Morris water maze. J Pharmacol Exp Ther 301: 915-924.

Walker DL, Ressler KJ, Lu KT, Davis M (2002). Facilitation of conditioned fear extinction by systemic administration or intraamygdala infusions of D-cycloserine as assessed with fearpotentiated startle in rats. J Neurosci 22: 2343-2351.

Walker JM, Huang SM, Strangman NM, Tsou K, Sanudo-Pena MC (1999). Pain modulation by release of the endogenous cannabinoid anandamide. Proc Natl Acad Sci USA 96: 12198-12203.

Witkin JM, Tzavara ET, Davis RJ, Li X, Nomikos GG (2005). A therapeutic role for cannabinoid $\mathrm{CB} 1$ receptor antagonists in major depressive disorders. Trends Pharmacol Sci 26: 609-617. 